Pacific

Journal of

Mathematics

\title{
MIXED NORM ESTIMATES FOR SOME AVERAGING OPERATORS IN $\mathbb{R}^{2}$
}

DANIEL M. OBERLIN

Volume $223 \quad$ No. 2

February 2006 


\title{
MIXED NORM ESTIMATES FOR SOME AVERAGING OPERATORS IN $\mathbb{R}^{2}$
}

\author{
DANIEL M. OBERLIN
}

\begin{abstract}
We obtain mixed norm estimates for averaging operators on $\mathbb{R}^{2}$ defined by convolution with $\mu$, where either $\mu$ is arclength measure on certain curves in $\mathbb{R}^{2}$ or $d \mu=d x / x^{\beta}$ on the positive $x$-axis.
\end{abstract}

\section{Introduction}

Suppose $\mu$ is a positive Borel measure on $\mathbb{R}^{2}$ and $\theta \in[0,2 \pi]$. Write $\mu_{\theta}$ for the measure obtained by rotating $\mu$ through the angle $\theta$ around the origin. For suitable functions $f$ on $\mathbb{R}^{2}$, define the operator $T$ by $T f(x, \theta)=f * \mu_{\theta}(x)$ for $x \in \mathbb{R}^{2}$. This paper is concerned with $L^{p} \rightarrow L^{q}\left(L^{s}\right)$ estimates of the form

$$
\|\| T f\left\|_{L^{s}([0,2 \pi])}\right\|_{L^{q}\left(\mathbb{R}^{2}\right)} \leq C\|f\|_{L^{p}\left(\mathbb{R}^{2}\right)}
$$

for some $C=C(p, q, s, T)$ when either $\mu$ is arclength measure on certain curves in $\mathbb{R}^{2}$ or $d \mu$ is $d x_{1} / x_{1}^{\beta}$ on the positive $x_{1}$-axis. Our motivation in the first case is the paper [Ricci and Travaglini 2001], which deals with mixed norm estimates with the $\theta$ norm on the outside. In the second case it is the paper [Christ et al. 1986] of Christ, Duoandikoetxea, and Rubio de Francia, which gives (in $n$ dimensions) $L^{p} \rightarrow L^{p}\left(L^{s}\right)$ estimates for operators like $T$ but with fractional integration replaced by a maximal function or Hilbert transform along the $x_{1}$-axis. Our main tool, a particular application of the Sobolev embedding theorem, is borrowed from [Christ et al. 1986].

We begin by recording necessary conditions for (1) to hold in a very simple case: abusing notation, write $\theta$ for $(\cos \theta, \sin \theta) \in \mathbb{R}^{2}$ and consider the operator

$$
T f(x, \theta)=\int_{0}^{1} f(x-t \theta) d t .
$$

MSC2000: 42B20.

Keywords: mixed norm, averaging operator. 
Assume that (1) holds and, as usual, test (1) when $f=\chi_{E}$. Choosing for $E$ an eccentric rectangle $[0,1] \times[0, \delta]$ shows that

$$
\frac{1}{p} \leq \frac{1}{q}+\frac{1}{s}
$$

Choosing $E$ to be a large ball gives

$$
\frac{1}{q} \leq \frac{1}{p}
$$

If $E$ is a small ball of radius $\delta$ then the fact that $T f(x, \theta) \approx \delta$ for $x$ in a set of measure $\approx \delta^{2}$ and for all $\theta$ gives

$$
\frac{1}{p} \leq \frac{1}{2}+\frac{1}{q}
$$

while $T f(x, \theta) \approx \delta$ for $\theta$ in a set of measure $\approx \delta /|x|$ if $2 \delta \leq|x| \leq 1$ leads to

$$
\frac{2}{p} \leq 1+\frac{1}{s}
$$

and to

$$
\frac{2}{p}<1+\frac{1}{s} \text { if } \frac{1}{s}=\frac{2}{q} .
$$

Our first main result shows that these necessary conditions are very nearly sufficient when $\mu$ is (equivalent to) arclength measure on certain convex curves in $\mathbb{R}^{2}$.

Theorem 1. Suppose that $u$ is continuously differentiable and convex on an interval $[a, b]$ containing 0 . Suppose also that the signs of $u(t) u^{\prime}(t)$ and $t$ agree on $[a, b]$ (which will be the case if, for example, $u \geq 0$ and $u^{\prime}(0)=0$ ). Let $d \mu$ be the measure $d t$ on the graph of $u$ over $[a, b]$. Suppose $p, q, s \in(1, \infty)$ satisfy the inequalities

$$
0 \leq \frac{1}{p}-\frac{1}{q} \leq \frac{1}{2}, \quad \frac{1}{s}>\max \left\{\frac{1}{p}-\frac{1}{q}, \frac{2}{p}-1\right\} .
$$

Then (1) holds.

The proof of this theorem, given in Section 2, will show that certain endpoint results are also valid.

Next define, for $-1 \leq \beta<1$ and functions $f$ on $\mathbb{R}^{2}$,

$$
I_{\beta} f(x, \theta)=\int_{0}^{\infty} f(x-t \theta) \frac{d t}{t^{\beta}} .
$$

Theorem 2. If (1) holds for $T=I_{\beta}$, then

$$
\frac{1}{p}-\frac{1}{q}=\frac{1-\beta}{2}, \quad \frac{1}{s}>\frac{2}{p}-1, \quad \frac{1}{s} \geq \frac{1-\beta}{2} .
$$


Conversely, if these conditions hold for $p, q \in(1, \infty)$ and with strict inequality in the last, then (1) holds for $T=I_{\beta}$.

The proof is given in Section 3, which also contains a corollary to the theorem. As in [Christ et al. 1986], it is possible to obtain $n$-dimensional extensions of Theorem 2. But the situation is much more complicated in higher dimensions and the results we have there are far from sharp. So we have chosen to limit the scope of this article to the case $n=2$.

\section{Proof of Theorem 1}

The proof is by interpolation. Of the various endpoint results which will be required, the following is most interesting.

Lemma 1. Suppose that $\frac{1}{2} \leq \frac{1}{p} \leq \frac{3}{4}$, that $\frac{1}{p}+\frac{1}{q}=1$, and that $\frac{1}{s}>\frac{1}{p}-\frac{1}{q}=\frac{2}{p}-1$. Then the conclusion of Theorem 1 holds.

The technical tool required for the proof of Lemma 1 is an analog of a lemma of Podkorytov [1991], which also figured in the proof in [Ricci and Travaglini 2001].

Lemma 2. Suppose that $u$ is continuously differentiable and convex on an interval I. Suppose that $\psi$ is either an appropriate cutoff function on I or $\chi_{I}$. Define, for $\rho>0$,

$$
k_{\rho}(\theta)=\int e^{-2 \pi i \rho(t \cos \theta+u(t) \sin \theta)} \psi(t) d t .
$$

Then $k_{\rho}$ satisfies the following Sobolev norm estimate uniformly in $\rho$ :

$$
\left\|k_{\rho}\right\|_{L_{1 / 2}^{2}(\theta)} \leq C(u, \psi) .
$$

Proof of Lemma 2. Podkorytov's lemma is the statement that

$$
\left\|k_{\rho}\right\|_{L^{2}(\theta)} \leq \frac{C(u, \psi)}{(1+|\rho|)^{1 / 2}} .
$$

We will follow the proof of that lemma given in [Brandolini et al. 2001] to observe that also

$$
\left\|\frac{d}{d \theta} k_{\rho}\right\|_{L^{2}(\theta)} \leq C(u, \psi)(1+|\rho|)^{1 / 2} .
$$

Interpolation of these two estimates proves Lemma 2.

We note, for future reference, that the constants $C(u, \psi)$ in Podkorytov's lemma and in (2) depend on $u$ only through the $L^{\infty}$ norms of $u$ and $u^{\prime}$ on $[a, b]$.

Writing

$$
\gamma(\theta, t)=u(t) \cos \theta-t \sin \theta
$$


we have

$$
\frac{d}{d \theta} k_{\rho}(\theta)=-2 \pi i \rho \int \gamma(\theta, t) e^{-2 \pi i \rho(t \cos \theta+u(t) \sin \theta)} \psi(t) d t
$$

and so

$$
\begin{aligned}
& \int_{0}^{2 \pi}\left|\frac{d}{d \theta} k_{\rho}(\theta)\right|^{2} d \theta \\
& \quad=4 \pi^{2} \rho^{2} \int_{0}^{2 \pi} \iint \gamma\left(\theta, t_{1}\right) \gamma\left(\theta, t_{2}\right) e^{-2 \pi i \rho\left(\left(t_{1}-t_{2}\right) \cos \theta+\left(u\left(t_{1}\right)-u\left(t_{2}\right)\right) \sin \theta\right)} \\
& \times \psi\left(t_{1}\right) \psi\left(t_{2}\right) d t_{1} d t_{2} d \theta .
\end{aligned}
$$

Now $\gamma\left(\theta, t_{1}\right) \gamma\left(\theta, t_{2}\right)$ is a linear combination of four terms $T_{j}\left(\theta, t_{1}, t_{2}\right)$, like

$$
T_{2}\left(\theta, t_{1}, t_{2}\right)=u\left(t_{1}\right) \cos (\theta) t_{2} \sin (\theta) .
$$

To establish (2) it is enough to show that

$$
\begin{array}{r}
\left|\iiint_{0}^{2 \pi} T_{j}\left(\theta, t_{1}, t_{2}\right) e^{-2 \pi i \rho\left(\left(t_{1}-t_{2}\right) \cos \theta+\left(u\left(t_{1}\right)-u\left(t_{2}\right)\right) \sin \theta\right)} d \theta \psi\left(t_{1}\right) \psi\left(t_{2}\right) d t_{1} d t_{2}\right| \\
\leq \frac{C(u, \psi)}{1+|\rho|}
\end{array}
$$

for each $j$. The case $j=2$ is typical. If

$$
\sin \phi=\frac{t_{1}-t_{2}}{\sqrt{\left(t_{1}-t_{2}\right)^{2}+\left(u\left(t_{1}\right)-u\left(t_{2}\right)\right)^{2}}}, \quad \cos \phi=\frac{u\left(t_{1}\right)-u\left(t_{2}\right)}{\sqrt{\left(t_{1}-t_{2}\right)^{2}+\left(u\left(t_{1}\right)-u\left(t_{2}\right)\right)^{2}}},
$$

then the inner integral is

$$
u\left(t_{1}\right) t_{2} \int_{0}^{2 \pi} \cos (\theta+\phi) \sin (\theta+\phi) e^{-2 \pi i \rho \sqrt{\left(t_{1}-t_{2}\right)^{2}+\left(u\left(t_{1}\right)-u\left(t_{2}\right)\right)^{2}} \cos \theta} d \theta
$$

This is again a linear combination of four terms, of which

$u\left(t_{1}\right) t_{2} \frac{\left(u\left(t_{1}\right)-u\left(t_{2}\right)\right)^{2}}{\left(t_{1}-t_{2}\right)^{2}+\left(u\left(t_{1}\right)-u\left(t_{2}\right)\right)^{2}} \int_{0}^{2 \pi} \cos \theta \sin \theta e^{-2 \pi i \rho \sqrt{\left(t_{1}-t_{2}\right)^{2}+\left(u\left(t_{1}\right)-u\left(t_{2}\right)\right)^{2}} \cos \theta} d \theta$ is typical. The integral in this expression is a linear combination of Bessel functions

$$
J_{m}\left(2 \pi \rho \sqrt{\left(t_{1}-t_{2}\right)^{2}+\left(u\left(t_{1}\right)-u\left(t_{2}\right)\right)^{2}}\right)
$$

for $m=-2,0,2$, and so it is enough to write

$$
v\left(t_{1}, t_{2}\right)=\frac{\left(u\left(t_{1}\right)-u\left(t_{2}\right)\right)^{2}}{\left(t_{1}-t_{2}\right)^{2}+\left(u\left(t_{1}\right)-u\left(t_{2}\right)\right)^{2}}
$$


and then to show that

$$
\begin{array}{r}
\left|\iint u\left(t_{1}\right) t_{2} v\left(t_{1}, t_{2}\right) J_{m}\left(2 \pi \rho \sqrt{\left(t_{1}-t_{2}\right)^{2}+\left(u\left(t_{1}\right)-u\left(t_{2}\right)\right)^{2}}\right) \psi\left(t_{1}\right) \psi\left(t_{2}\right) d t_{1} d t_{2}\right| \\
\leq \frac{C(u, \psi)}{1+|\rho|} .
\end{array}
$$

To obtain this inequality we essentially repeat (for the reader's convenience) part of the proof of [Brandolini et al. 2001, Lemma 2]. By splitting the original curve if necessary we can assume that $u^{\prime}(t)$ is of one sign on $[a, b]$. Restricting, without loss of generality, to the range $t_{1} \geq t_{2}$, we change variables in the inner integral by setting

$$
z=z\left(t_{1}, t_{2}\right)=\sqrt{\left(t_{1}-t_{2}\right)^{2}+\left(u\left(t_{1}\right)-u\left(t_{2}\right)\right)^{2}} .
$$

A calculation shows that

$$
\frac{\partial z}{\partial t_{1}}=\frac{1+\frac{u\left(t_{1}\right)-u\left(t_{2}\right)}{t_{1}-t_{2}} u^{\prime}\left(t_{1}\right)}{\sqrt{1+\left(\frac{u\left(t_{1}\right)-u\left(t_{2}\right)}{t_{1}-t_{2}}\right)^{2}}}
$$

It follows from the convexity of $u$ that $\partial z / \partial t_{1}$ is a quotient of monotone functions of $t_{1}$ (which will be needed later) and also that

$$
\frac{1}{\sqrt{1+\left\|u^{\prime}\right\|_{L^{\infty}([a, b])}^{2}}} \leq\left|\frac{\partial z}{\partial t_{1}}\right| \leq 1+\left\|u^{\prime}\right\|_{L^{\infty}([a, b])}^{2} .
$$

Setting

$$
\omega\left(z, t_{2}\right)=\left|\frac{\partial t_{1}}{\partial z}\right|
$$

we have

$$
\begin{array}{r}
\iint u\left(t_{1}\right) t_{2} v\left(t_{1}, t_{2}\right) J_{m}\left(2 \pi \rho \sqrt{\left(t_{1}-t_{2}\right)^{2}+\left(u\left(t_{1}\right)-u\left(t_{2}\right)\right)^{2}}\right) \psi\left(t_{1}\right) \psi\left(t_{2}\right) d t_{1} d t_{2} \\
=\iint u\left(t_{1}\left(z, t_{2}\right)\right) v\left(t_{1}\left(z, t_{2}\right), t_{2}\right) J_{m}(2 \pi \rho z) \psi\left(t_{1}\left(z, t_{2}\right)\right) \omega\left(z, t_{2}\right) d z t_{2} \psi\left(t_{2}\right) d t_{2} \\
=\rho^{-1} \iint u\left(t_{1}\left(w / \rho, t_{2}\right)\right) v\left(t_{1}\left(w / \rho, t_{2}\right), t_{2}\right) J_{m}(2 \pi w) \psi\left(t_{1}\left(w / \rho, t_{2}\right)\right) \\
\omega\left(w / \rho, t_{2}\right) d w t_{2} \psi\left(t_{2}\right) d t_{2},
\end{array}
$$

where $t_{1}\left(z, t_{2}\right)$ is the inverse of the change of variables $t_{1} \mapsto z$. To bound the last integral, note first that the convexity of $u$ implies the monotonicity of

$$
\frac{u\left(t_{1}\right)-u\left(t_{2}\right)}{t_{1}-t_{2}}
$$


and so of $v\left(t_{1}, t_{2}\right)$ as a function of $t_{1}$; secondly, that

$$
\left|\int_{0}^{w} J_{m}(2 \pi s) d s\right| \leq c
$$

and then apply the following easily-established lemma:

Lemma. Suppose that $\alpha_{j}(t)$, for $1 \leq j \leq J$, are monotone functions on $[c, d]$ and that $\beta(t)$ is such that

$$
\left|\int_{c}^{t} \beta(s) d s\right| \leq c
$$

for some $c$ and all $t \in[c, d]$. Then

$$
\left|\int_{c}^{d} \prod_{j=1}^{J} \alpha_{j}(w) \beta(w) d w\right| \leq 3^{J} c \prod_{j=1}^{J}\left\|\alpha_{j}\right\|_{L^{\infty}([c, d])} .
$$

Proof of Lemma 1. First, Lemma 2 implies the estimate

$$
\|\| T f\left\|_{L_{1 / 2}^{2}(\theta)}\right\|_{L^{2}\left(\mathbb{R}^{2}\right)} \leq C\|f\|_{L^{2}\left(\mathbb{R}^{2}\right)} .
$$

One way to check this is by observing that if the kernel $K_{n}$ on $\mathbb{R}^{2}$ satisfies

$$
K_{n} * f(x)=\widehat{T f(x, \cdot)}(n) \quad\left(=\frac{1}{2 \pi} \int_{0}^{2 \pi} T f(x, \theta) e^{-i n \theta} d \theta\right),
$$

then

$$
\widehat{K}_{n}(y)=\frac{1}{2 \pi} \int_{0}^{2 \pi} \int e^{-2 \pi i y \cdot x} d \mu_{\theta}(x) e^{-i n \theta} d \theta
$$

and so Lemma 2 is essentially the statement that

$$
\sum_{n=-\infty}^{\infty}\left|\widehat{K}_{n}(y)\right|^{2}(1+|n|) \leq C
$$

uniformly for $y \in \mathbb{R}^{2}$.

Since (3) implies an $L^{2} \rightarrow L^{2}(B M O)$ estimate for $T$, it is natural to think of interpolating that estimate with an $L^{1} \rightarrow L^{\infty}\left(L^{1}\right)$ estimate for $T$ (though this would lead to Lemma 1 without the restriction $\left.\frac{1}{p} \leq \frac{3}{4}\right)$. Such an $L^{1} \rightarrow L^{\infty}\left(L^{1}\right)$ estimate would be equivalent to the inequality

$$
\begin{aligned}
\int_{0}^{2 \pi} \int_{a}^{b} f(t \cos \theta+u(t) \sin \theta,-t \sin \theta+u(t) \cos \theta) \psi(t) d t & d \theta \\
& \leq C \int_{\mathbb{R}^{2}} f(x) d x
\end{aligned}
$$

for nonnegative measurable $f$ on $\mathbb{R}^{2}$. The absolute value of the Jacobian of

$$
(\theta, t) \mapsto(t \cos \theta+u(t) \sin \theta,-t \sin \theta+u(t) \cos \theta)
$$


is $\left|t+u(t) u^{\prime}(t)\right|$. Since $\left|t+u(t) u^{\prime}(t)\right| \geq|t|$ because of the hypotheses on $u$, (4) will hold if 0 is not in the support of $\psi$, but may fail otherwise. It follows by interpolation that Lemma 1 holds (and for $\frac{1}{2} \leq \frac{1}{p} \leq 1$ ) if 0 is not in the support of $\psi$. Thus we may assume for the remainder of the proof of Lemma 1 that 0 belongs to the support of $\psi$. The idea now is to consider an analytic family defined by

$$
T_{z} f(x, \theta)=\int f(x-(t \cos \theta+u(t) \sin \theta,-t \sin \theta+u(t) \cos \theta))|t|^{z} \psi(t) d t,
$$

though, for technical reasons, we shall actually consider the related family

$$
T_{z} f(x, \theta)=\sum_{n=0}^{\infty} 2^{-n z} T_{n} f(x, \theta),
$$

where

$$
T_{n} f(x, \theta)=\int f(x-(t \cos \theta+u(t) \sin \theta,-t \sin \theta+u(t) \cos \theta)) \phi\left(2^{n}|t|\right) d t
$$

for some appropriate cutoff function $\phi$ supported away from 0 . If $z=1+i$ s then the inequality $\left|t+u(t) u^{\prime}(t)\right| \geq|t|$ between $|t|$ and the Jacobian of (5) shows that $T_{z}: L^{1} \rightarrow L^{\infty}\left(L^{1}\right)$ with a norm bound which is independent of $s$. Since $T \approx T_{0}$, Lemma 1 will follow from interpolation if we establish that $T_{z}: L^{2} \rightarrow L^{2}(B M O)$ uniformly in $s$ whenever $z=-1+\epsilon+i s$ for small $\epsilon>0$. And this will be a consequence of the estimate

$$
\|\| T_{n}\left\|_{L_{1 / 2}^{2}(\theta)}\right\|_{L^{2}\left(\mathbb{R}^{2}\right)} \leq \frac{C\|f\|_{L^{2}\left(\mathbb{R}^{2}\right)}}{2^{n}} .
$$

Letting

$$
k_{\rho, n}(\theta)=\int e^{-2 \pi i \rho(t \cos \theta+u(t) \sin \theta)} \phi\left(2^{n}|t|\right) d t,
$$

inequality (6) will follow (as in the beginning of this proof) from the estimate, uniform in $\rho$,

$$
\left\|k_{\rho, n}\right\|_{L_{1 / 2}^{2}(\theta)} \leq 2^{-n} C
$$

To see (7) write

$$
\begin{aligned}
\tilde{k}_{\rho, n}(\theta) & =\int e^{-2 \pi i \rho(t \cos \theta+(u(t)-u(0)) \sin \theta)} \phi\left(2^{n}|t|\right) d t \\
& =2^{-n} \int e^{-2 \pi i \rho 2^{-n}\left(s \cos \theta+2^{n}\left(u\left(s / 2^{n}\right)-u(0)\right) \sin \theta\right)} \phi(|s|) d s .
\end{aligned}
$$

If

$$
u_{n}(s)=2^{n}\left(u\left(s / 2^{n}\right)-u(0)\right)
$$


then $\left|u_{n}\right|$ and $\left|u_{n}{ }^{\prime}\right|$ are bounded on the support of $\phi$ by

$$
C(\phi)\left\|u_{n}^{\prime}\right\|_{L^{\infty}([a, b])} .
$$

The remark concerning uniformity in the proof of Lemma 2 now shows that

$$
\left\|\tilde{k}_{\rho, n}\right\|_{L^{2}(\theta)} \leq \frac{C(u, \psi)}{2^{n}(1+|\rho|)^{1 / 2}}
$$

and

$$
\left\|\frac{d}{d \theta} \tilde{k}_{\rho, n}\right\|_{L^{2}(\theta)} \leq \frac{C(u, \psi)(1+|\rho|)^{1 / 2}}{2^{n}} .
$$

Since

$$
k_{\rho, n}(\theta)=e^{-2 \pi i \rho u(0) \sin \theta} \tilde{k}_{\rho, n}(\theta),
$$

similar inequalities hold for $k_{\rho, n}$. Interpolating these inequalities gives (7), completing the proof of Lemma 1 .

To complete the proof of Theorem 1 it is enough, by interpolation, to show that the conclusion holds whenever either $\frac{1}{p}-\frac{1}{q}=0$ or $\frac{1}{p}-\frac{1}{q}=\frac{1}{2}$. For the first case, recall that Lemma 1 gives an $L^{2} \rightarrow L^{2}\left(L^{s}\right)$ estimate for $s<\infty$. Interpolating this with the obvious $L^{1} \rightarrow L^{1}\left(L^{1}\right)$ and $L^{\infty} \rightarrow L^{\infty}\left(L^{\infty}\right)$ estimates provides the desired result. For the second case, $\frac{1}{p}-\frac{1}{q}=\frac{1}{2}$, the argument splits depending on whether $\frac{1}{2}<\frac{1}{p} \leq \frac{3}{4}$ or $\frac{3}{4} \leq \frac{1}{p}<1$. For $\frac{1}{2}<\frac{1}{p}^{q} \leq \frac{3}{4}$ we begin by noting that if $E \subseteq \mathbb{R}^{2}$ is a Borel set, then, for any $x \in \mathbb{R}^{2}$,

$$
\begin{aligned}
\int_{0}^{2 \pi} & \left(T \chi_{E}(x, \theta)\right)^{2} d \theta \\
& =\int_{0}^{2 \pi}\left(\int \chi_{E}(x-(t \cos \theta+u(t) \sin \theta,-t \sin \theta+u(t) \cos \theta)) \psi(t) d t\right)^{2} d \theta .
\end{aligned}
$$

Since

$$
\left(\int \chi_{F}(t) d t\right)^{2} \leq 4 \int \chi_{F}(t)|t| d t
$$

for measurable $F \subseteq \mathbb{R}$, it follows that

$$
\begin{aligned}
& \int_{0}^{2 \pi}\left(T \chi_{E}(x, \theta)\right)^{2} d \theta \\
& \leq C \int_{0}^{2 \pi} \int_{a}^{b} \chi_{E}(x-(t \cos \theta+u(t) \sin \theta,-t \sin \theta+u(t) \cos \theta))|t| d t d \theta \\
& \leq C \int_{0}^{2 \pi} \int_{a}^{b} \chi_{E}(x-(t \cos \theta+u(t) \sin \theta,-t \sin \theta+u(t) \cos \theta)) \\
& \leq C|E| . \times\left|t+u(t) u^{\prime}(t)\right| d t d \theta \\
&
\end{aligned}
$$


We interpret this as an $L^{2,1} \rightarrow L^{\infty}\left(L^{2}\right)$ estimate which implies an $L^{2,1} \rightarrow L^{\infty}\left(L^{s}\right)$ estimate if $1 \leq s<2$. Lemma 1 gives an $L^{4 / 3} \rightarrow L^{4}\left(L^{s}\right)$ estimate for the same values of $s$, and so interpolation gives an $L^{p} \rightarrow L^{q}\left(L^{s}\right)$ estimate whenever $\frac{1}{p}-\frac{1}{q}=\frac{1}{2}$, $\frac{4}{3} \leq p<2$, and $1 \leq s<2$. For $\frac{3}{4} \leq \frac{1}{p}<1$, let the positive kernel $K(x)$ on $\mathbb{R}^{2}$ be defined so that

$$
\int_{0}^{2 \pi} T f(x, \theta) d \theta=f * K(x)
$$

for appropriate $f$ on $\mathbb{R}^{2}$. As we will observe below, $K \in L^{2, \infty}\left(\mathbb{R}^{2}\right)$. This implies an $L^{p} \rightarrow L^{q}\left(L^{1}\right)$ estimate whenever $\frac{1}{p}-\frac{1}{q}=\frac{1}{2}$. That estimate can be interpolated with the aforementioned $L^{4 / 3} \rightarrow L^{4}\left(L^{s}\right)$ estimate for $1 \leq s<2$ to provide the required $L^{p} \rightarrow L^{q}\left(L^{s}\right)$ estimate whenever $\frac{1}{p}-\frac{1}{q}=\frac{1}{2}, \frac{3}{4} \leq \frac{1}{p}<1$, and $1 \leq s<p /(2-p)$. Finally, to see that $K \in L^{2, \infty}\left(\mathbb{R}^{2}\right)$, observe that for Borel $E \subseteq \mathbb{R}^{2}$ we have

$$
\begin{aligned}
& \int_{E} K(x) d x \\
& \quad=\int_{0}^{2 \pi} \int_{a}^{b} \frac{\chi_{E}(t \cos \theta+u(t) \sin \theta,-t \sin \theta+u(t) \cos \theta) \psi(t)}{\left|t+u(t) u^{\prime}(t)\right|}\left|t+u(t) u^{\prime}(t)\right| d t d \theta .
\end{aligned}
$$

Since $\left|t+u(t) u^{\prime}(t)\right|$ is the Jacobian of (5), it is enough to note that, for $\lambda>0$ and since $|t| \leq\left|t+u(t) u^{\prime}(t)\right|$,

$$
\int_{\left\{1 /\left|t+u(t) u^{\prime}(t)\right| \geq \lambda\right\}}\left|t+u(t) u^{\prime}(t)\right| d t \leq \int_{\{|t| \leq 1 / \lambda\}} \frac{1}{\lambda} d t=\frac{2}{\lambda^{2}} .
$$

This completes the proof of Theorem 1 .

Perhaps surprisingly, the analog of Theorem 1 fails for the function $u(t)=$ $1-t^{2} / 2:$ if

$$
A_{\delta}=\{1-\delta \leq|x| \leq 1\} \text { and } f=\chi_{A_{\delta}}
$$

then $T f(x, \theta) \approx \delta^{1 / 4}$ for $|x| \leq \delta / 10$ and (1) taken together lead to $1+\frac{8}{q} \geq \frac{4}{p}$, an inequality violated by some triples $(p, q, s)$ satisfying the hypotheses of Theorem 1.

\section{Proof of Theorem 2}

The necessary conditions are easily established: the first is a consequence of the homogeneity of the operator $I_{\beta}$, the second and third follow by testing on the indicator function of a ball and of an eccentric rectangle.

The remainder of the proof is also somewhat parallel to the proof of Theorem 1. We begin with a lemma analogous to Lemma 1.

Lemma 3. Suppose that $\frac{1}{2}<\frac{1}{p} \leq 1$, that $\frac{1}{p}+\frac{1}{q}=1$, and that $\frac{1}{s}>\frac{2}{p}-1 \doteq \frac{1-\beta}{2}$. Then the conclusion of Theorem 2 holds. 
Proof. When $p=1$ the lemma holds trivially and with $s=1$. So, by interpolation, it is enough to prove the lemma when $p$ is close to 2 . Recall that $\theta$, by abuse of notation, will sometimes mean $(\cos \theta, \sin \theta)$. We fix a suitable $f$ and calculate

$$
\begin{aligned}
2 \pi \widehat{\left.I_{\beta} \widehat{f(x,} \cdot\right)(n)} & =\int_{0}^{2 \pi} I_{\beta} f(x, \theta) e^{-i n \theta} d \theta \\
& =\int_{0}^{2 \pi} \int_{0}^{\infty} f(x-t \theta) e^{-i n \theta} \frac{d t}{t^{\beta}} d \theta=f * K_{n}(x)
\end{aligned}
$$

if

Now

$$
K_{n}(t \theta)=\frac{e^{-i n \theta}}{t^{\beta+1}}
$$

$$
\begin{aligned}
\widehat{K}_{n}(y) \doteq \widehat{K}_{n}(|y| \phi) & =\int_{0}^{2 \pi} \int_{0}^{\infty} e^{-i n \theta} e^{-2 \pi i|y| t \cos (\theta-\phi)} \frac{d t}{t^{\beta}} d \theta \\
& =e^{-i n \phi} \int_{0}^{2 \pi} e^{-i n \theta} \int_{0}^{\infty} e^{-2 \pi i|y| t \cos \theta} \frac{d t}{t^{\beta}} d \theta
\end{aligned}
$$

Since

$$
\int_{0}^{\infty} e^{-2 \pi i \rho t} \frac{d t}{t^{\beta}}=c \rho^{\beta-1}
$$

for $\rho>0$, the observation

$$
\left|\int_{0}^{2 \pi}(\cos \theta)^{\beta-1} e^{-i n \theta} d \theta\right|=O\left(|n|^{-\beta}\right)
$$

gives

$$
\left|\widehat{K}_{n}(y)\right|=O\left(|y|^{\beta-1} n^{-\beta}\right) .
$$

The method of $T T^{*}$ shows that if $\frac{1}{p}+\frac{1}{q}=1$, if $m(y)$ is a nonnegative $L^{p} \rightarrow L^{q}$ Fourier multiplier, and if the measurable function $n$ satisfies $|n(y)| \leq m(y)$, then $n$ is also an $L^{p} \rightarrow L^{q}$ Fourier multiplier, and with multiplier norm bounded by that of $m$. Thus (8) and the fractional integration theorem show that

$$
\left\|K_{n}\right\|_{p, q}=O\left(n^{-\beta}\right),
$$

where the norm is that of the convolution operator with kernel $K_{n}$ from $L^{p}\left(\mathbb{R}^{2}\right)$ into $L^{q}\left(\mathbb{R}^{2}\right)$. Now, for $\gamma>0$,

$$
\begin{aligned}
\|\| I_{\beta} f\left\|_{L_{\gamma}^{2}(\theta)}\right\|_{L^{q}\left(\mathbb{R}^{2}\right)} & =\left\|\left(\sum\left(\left|K_{n} * f\right|(1+|n|)^{\gamma}\right)^{2}\right)^{1 / 2}\right\|_{L^{q}\left(\mathbb{R}^{2}\right)} \\
& \leq\left(\sum\left(\left\|K_{n} * f\right\|_{L^{q}\left(\mathbb{R}^{2}\right)}(1+|n|)^{\gamma}\right)^{2}\right)^{1 / 2} \\
& \leq\|f\|_{L^{p}\left(\mathbb{R}^{2}\right)}\left(\sum\left(\left\|K_{n}\right\|_{p, q}(1+|n|)^{\gamma}\right)^{2}\right)^{1 / 2} .
\end{aligned}
$$


By (8), the sum in the last inequality will be finite whenever $\gamma-\beta<-\frac{1}{2}$. The Sobolev embedding theorem thus yields an $L^{p} \rightarrow L^{q}\left(L^{s}\right)$ estimate for $I_{\beta}$ whenever $\frac{1}{2}-\frac{1}{s}<\beta-\frac{1}{2}$ or $\frac{1}{s}>1-\beta$. To replace $1-\beta$ by the $\frac{1-\beta}{2}$ in the statement of Lemma 3 requires another interpolation: noting that $\frac{1}{p}=\frac{3-\beta}{4}$, fix a point

$$
P_{0}=\left(\frac{1}{p_{0}}, \frac{1}{s_{0}}\right)
$$

in $\mathscr{Y} \doteq\left\{\left(\frac{1}{p}, \frac{1}{s}\right): 0 \leq \frac{1}{p}, \frac{1}{s} \leq 1\right\}$ such that

$$
\frac{1}{p_{0}}=\frac{3-\beta_{0}}{4}, \quad 1-\beta_{0} \geq \frac{1}{s_{0}}>\frac{1-\beta_{0}}{2}, \quad \beta_{0}>0 .
$$

(We may assume $\beta_{0}>0$ after an interpolation with the trivial case $\beta=-1$.) Let $L$ be the line segment in $\mathscr{S}$ parametrized by

$$
\frac{1}{p}=\frac{3-\beta}{4}, \quad \frac{1}{s}=1-\beta
$$

and choose a point $P$ above $L$ so that the line $L^{\prime}$ through $P$ and $(1,1)$ passes below $P_{0}$. Note that the conclusion of Lemma 3 holds if the point $\left(\frac{1}{p}, \frac{1}{s}\right)$ lies on $L^{\prime}$ and let $Q_{1}$ be the point on $L^{\prime}$ directly below $P_{0}$. Next choose $Q_{2}$ directly above $P_{0}$ and above $L$ (so that the conclusion of Lemma 3 holds at $Q_{2}$ ). Interpolating between the ( $p, q, s)$ points corresponding to $Q_{1}$ and $Q_{2}$ shows that Lemma 3 holds at $P_{0}$, completing the proof of Lemma 3.

To complete the proof of Theorem 2, it is enough (by interpolation with the trivial case $\beta=-1$ ) to show that the conclusion of Theorem 2 holds when $\beta>0$. So fix such a $\beta$. Fix $\frac{1}{p} \in\left(\frac{1-\beta}{2}, 1\right)$ and $q$ with $\frac{1}{p}-\frac{1}{q}=\frac{1-\beta}{2}$. There are two cases to considering, depending on whether $\frac{1}{p}<\frac{3-\beta}{4}$ or $\frac{1}{p}>\frac{3-\beta}{4}$ (the case $\frac{1}{p}=\frac{3-\beta}{4}$ being the subject of Lemma 3).

To deal with the first case begin by noting that, for Borel sets $E \subseteq \mathbb{R}^{2}$,

$$
\int_{0}^{2 \pi}\left(\int_{0}^{\infty} \chi_{E}(x+t \theta) \frac{d t}{t^{\beta}}\right)^{2 /(1-\beta)} d \theta \leq C(\beta) \int_{0}^{2 \pi} \int_{0}^{\infty} \chi_{E}(x+t \theta) t d t d \theta,
$$

since

$$
\left(\int_{F} \frac{d t}{t^{\beta}}\right)^{2 /(1-\beta)} \leq C(\beta) \int_{F} t d t
$$

for Borel $F \subseteq \mathbb{R}$. With $\frac{1}{p_{0}}=\frac{1-\beta}{2}$, we interpret (9) as an $L^{p_{0}, 1} \rightarrow L^{\infty}\left(L^{p_{0}}\right)$ estimate for $I_{\beta}$. This provides an $L^{p_{0}, 1} \rightarrow L^{\infty}\left(L^{s}\right)$ estimate whenever $\frac{1}{s}>\frac{1-\beta}{2}$ which can be interpolated with the estimate of Lemma 3 to provide the desired result.

For the second case, note that, so long as $\frac{1}{p}-\frac{1}{q}=\frac{1-\beta}{2}$, an $L^{p} \rightarrow L^{q}\left(L^{1}\right)$ estimate for $I_{\beta}$ follows from boundedness of the appropriate Riesz potential. Interpolating 
this with Lemma 3 shows that

$$
I_{\beta}: L^{p} \rightarrow L^{q}\left(L^{s}\right)
$$

whenever $\frac{1}{p}-\frac{1}{q}=\frac{1-\beta}{2}, \frac{3-\beta}{4} \leq \frac{1}{p}<1$ and $\frac{1}{s}>\frac{2}{p}-1$. This completes the proof of Theorem 2.

Analogously to [Christ et al. 1986, Corollary 4.2], the following result on operators with variable kernels is an easy consequence of Theorem 2.

Corollary. Suppose $1<p<q<\infty$ and $\frac{1}{p}-\frac{1}{q}=\frac{1-\beta}{2}$. Suppose the Borel kernel $K(x, y)$ on $\mathbb{R}^{2} \times \mathbb{R}^{2}$ satisfies the homogeneity condition

$$
K(x, \lambda y)=\lambda^{-1-\beta} K(x, y)
$$

for $\lambda>0$ and the growth condition

$$
\sup _{x \in \mathbb{R}^{2}}\|K(x, \cdot)\|_{L^{s^{\prime}}(\theta)}<\infty,
$$

where $s^{\prime}$ is the exponent dual to $s$ and the triple $(p, q, s)$ satisfies the hypotheses of Theorem 2. Define the operator $T$ by

$$
T f(x)=\int_{\mathbb{R}^{2}} f(x-y) K(x, y) d y .
$$

Then $T$ maps $L^{p}\left(\mathbb{R}^{2}\right)$ into $L^{q}\left(\mathbb{R}^{2}\right)$.

\section{References}

[Brandolini et al. 2001] L. Brandolini, A. Iosevich, and G. Travaglini, "Spherical means and the restriction phenomenon”, J. Fourier Anal. Appl. 7:4 (2001), 359-372. MR 2002e:42009 Zbl 0989. 42002

[Christ et al. 1986] M. Christ, J. Duoandikoetxea, and J. L. Rubio de Francia, "Maximal operators related to the Radon transform and the Calderón-Zygmund method of rotations", Duke Math. J. 53:1 (1986), 189-209. MR 88d:42032 Zbl 0656.42010

[Podkorytov 1991] A. N. Podkorytov, "On the asymptotic behaviour of the Fourier transform on a convex curve", Vestnik Leningrad. Univ. Mat. Mekh. Astronom. 1991:2 (1991), 50-57. In Russian; translation in Vestnik Leningrad Univ. Math. 24:2 (1991), 57-65. MR 93h:42019 Zbl 0741.42012

[Ricci and Travaglini 2001] F. Ricci and G. Travaglini, "Convex curves, Radon transforms and convolution operators defined by singular measures", Proc. Amer. Math. Soc. 129:6 (2001), 1739-1744. MR 2002i:42010 Zbl 0965.42007

Received May 5, 2004. Revised November 30, 2004.

DANIEL M. OBERLIN

Department of Mathematics

FLORIDA STATE UNIVERSITY

TALlahassee, FL 32306-4510

oberlin@math.fsu.edu 